\title{
A group- and smartphone-based psychological intervention to increase and maintain physical activity in patients with musculoskeletal conditions: study protocol for a randomized controlled trial ("MoVo-App")
}

\author{
Jiaxi Lin', Ramona Wurst², Sarah Paganini2 ${ }^{2}$, Vivien Hohberg², Stephan Kinkel ${ }^{3}$, Wiebke Göhner ${ }^{4}$,
} Christina Ramsenthaler ${ }^{2}$ and Reinhard Fuchs ${ }^{2}$

\begin{abstract}
Background: Interventions designed to increase the level of physical activity are crucial in the treatment of patients with musculoskeletal conditions. The psychological group-based intervention MoVo-LISA based on the Motivation-Volition (MoVo) Process Model has been shown to effectively promote physical activity. The aim of this study is to evaluate whether a MoVo-based app (MoVo-App) subsequent to MoVo-LISA during orthopedic inpatient care can support people to increase and maintain their amount of physical activity.

Methods/design: In this parallel-group randomized controlled trial, patients with musculoskeletal disorders will be randomized to either (a) a combination of the group-based intervention program MoVo-LISA to promote physical activity plus the MoVo-App or (b) the group-based intervention program alone without the app. The intervention group will receive the MoVo-App after discharge from inpatient rehabilitation. They receive help to increase and maintain their level of physical activity (initiated by the group program) by tracking their health goals, activity plans, major barriers, and barrier management that were developed during the group-based program. We will recruit 224 initially minimally active participants during orthopedic rehabilitation care. Outcomes are assessed at clinic admission; discharge; 6 weeks; and 3 (post-treatment), 6 , and 12 months after discharge (follow-up). The primary outcome is sport activity (active/inactive and minutes of activity) at 6-month follow-up. Secondary outcomes are movement activity, cognitive mediators of behavioral change (e.g., self-efficacy, action planning), and health-related variables (e.g., pain intensity, depression). To evaluate intervention effects, linear mixed effects models (both on intention-to-treat basis with an additional per-protocol analysis) will be conducted with each outcome variable and with time as the within-subjects factor and group as the between-subjects factor, along with all two-way interactions and accounting for covariates as fixed effects.
\end{abstract}

(Continued on next page)

* Correspondence: sarah.paganini@sport.uni-freiburg.de

${ }^{2}$ Department of Sport Psychology, Institute of Sports and Sport Science, University of Freiburg, Schwarzwaldstrasse 175, 79117 Freiburg, Germany

Full list of author information is available at the end of the article

(c) The Author(s). 2020 Open Access This article is licensed under a Creative Commons Attribution 4.0 International License, which permits use, sharing, adaptation, distribution and reproduction in any medium or format, as long as you give appropriate credit to the original author(s) and the source, provide a link to the Creative Commons licence, and indicate if changes were made. The images or other third party material in this article are included in the article's Creative Commons licence, unless indicated otherwise in a credit line to the material. If material is not included in the article's Creative Commons licence and your intended use is not permitted by statutory regulation or exceeds the permitted use, you will need to obtain permission directly from the copyright holder. To view a copy of this licence, visit http://creativecommons.org/licenses/by/4.0/ The Creative Commons Public Domain Dedication waiver (http://creativecommons.org/publicdomain/zero/1.0/) applies to the data made available in this article, unless otherwise stated in a credit line to the data. 
(Continued from previous page)

Discussion: This is the first evaluation of the effectiveness of an app in combination with a face-to-face group intervention to promote physical activity. The approach of using an app in addition to an effective face-to-face intervention program, both based on the Movo model, might sustain positive intervention effects introduced in routine health care.

Trial registration: The trial "A group- and smartphone-based psychological intervention to increase physical activity in patients with musculoskeletal conditions: A randomized controlled trial" is registered at the World Health Organization International Clinical Trials Registry Platform via the German Clinical Studies Trial Register (DRKS), DRKS00014814. Registered on 18 October 2018; URL: https://www.drks.de/drks_web/navigate.do?navigationld=trial. HTML\&TRIAL_ID=DRKS00014814.

Keywords: Blended intervention, App, Motivation and volition, Psychological intervention, Long term, Exercise, Sport activity, Orthopedic rehabilitation, Randomized controlled trial

\section{Background}

Musculoskeletal disorders such as chronic back pain, rheumatoid arthritis, and osteoporosis are highly prevalent conditions. The global prevalence of low back pain lasting longer than 1 month is estimated to be $23.2 \%$ [1]. The 1-year prevalence of back pain in Europe is $46.1 \%$, and for neck and upper limb pain, it is $44.6 \%$ [2]. Furthermore, approximately 54 million (22.7\%) of adults aged $\geq 18$ years were diagnosed with arthritis in the United States between 2010 and 2012 [3]. In Germany, the prevalence rates of back pain and osteoarthritis are $22.5 \%$ and $20.6 \%$, respectively, in people aged 20-75 [4]. Impairments of the musculoskeletal system cause high physical and economic burden to the individual and society $[5,6]$. Low back pain, one of the most prevalent chronic musculoskeletal conditions, is the leading cause of years lived with disability worldwide, with other musculoskeletal conditions among the top ten causes [7].

Interventions to increase and maintain physical activity are among the most effective interventions for preventing further disease symptoms such as pain and dysfunction, and promoting health-related quality of life in patients with musculoskeletal disorders [5, 8-10]. An overview of systematic reviews found that exercise therapy yielded standardized mean differences between 0.30 and 0.65 for improving pain and function in individuals with different musculoskeletal conditions [5]. However, few patients manage to establish a stable level of physical activity behavior after inpatient treatment under their own volition [11, 12]. The Motivation-Volition concept for promoting Lifestyle-Integrated Sport Activity (MoVo-LISA; www.movo-konzept.de), a group-based intervention administered during inpatient rehabilitation, was developed to overcome low exercise adherence and to effectively promote long-term physical activity $[13,14]$. Several randomized controlled trials (RCTs) (and quasi-experimental studies) have shown that MoVo-LISA has beneficial short- and long-term effects in patients with different conditions [15-19]. Promoting physical activity has consistently been shown to lead to a significant increase in exercise duration compared with preintervention or control group in psychosomatic [15], orthopedic [16, 17, 20], and cardiovascular [19] patients and in overweight or obese individuals [18]. In orthopedic patients, Fuchs and colleagues [16] reported the MoVo-LISA group as being more active than the usual care group by 28.5 min per week, even 12 months after discharge (per-protocol analyses). Moreover, investigations of treatment mediators strongly support the theoretical framework of the MoVo concept because behavior changes were shown to be based on changes in the underlying psychological factors $[17,21]$.

In previous studies, exercise duration measured after completion of MoVo-LISA was, on average, $156 \mathrm{~min} /$ week in the intervention group (IG). However, exercise duration decreased to $92 \mathrm{~min} /$ week at 6 months after discharge $[13,17]$. New effective and cost-effective ways to prevent this decrease are needed. One way to do so could be to use technologies such as mobile applications (apps) to support participants in maintaining MoVoLISA promoted habits and thus their level of physical activity. Several studies and systematic reviews with and without meta-analyses have shown that internet- and mobile app-based interventions can effectively and potentially cost-effectively increase and help maintain the amount of physical activity [12, 22-29]. A recent systematic review reported significant health improvements of app-based interventions targeting at physical activity in 14 of 21 studies [28].

Despite significant improvements, the positive effects of mobile apps have not been consistently reported, particularly in studies that use apps as stand-alone interventions. This might be due to internet- and mobile app-based interventions battling with repeatedly high dropout rates [30-35]. In internet-delivered physical activity behavior change programs, the average cross-study attrition rate was $23 \%$ in the IGs (reported in 28 of 34 studies) [29]. This effect seems to be more pronounced 
in studies using apps as stand-alone interventions. There is also evidence that multicomponent interventions lead to higher efficacy in behavioral and health outcomes than interventions consisting of apps alone [28]. Thus, blended interventions combining both face-to-face sessions and mobile material might be less prone to suffer from high withdrawal. Another advantage of blended interventions is that they can increase therapy intensity, which in turn might increase intervention effects [36]. In a systematic review of blended face-to-face and internet-based interventions for the treatment of mental disorders in adults, Erbe and colleagues found that these interventions can be more effective than no treatment [37]. Thus, an app embedded within a face-to-face intervention might help maintain the increased amount of physical activity that was acquired in a face-to-face intervention. Therefore, we developed a smartphone app based on the MoVo concept ("MoVo-App") and adapted it to the MoVo-LISA group intervention.

The following is the primary research question:

1. Can the combination of the face-to-face groupbased intervention MoVo-LISA and MoVo-App increase maintenance of the initiated physical activity level in patients with musculoskeletal disorders more than MoVo-LISA without subsequent MoVoApp use?

We hypothesize that the additional MoVo-App can help to maintain the initiated level of physical activity (by the face-to-face group program) 6 months after clinic discharge in patients with musculoskeletal disorders more than MoVo-LISA without the app.

The following are secondary research questions:

2. Can the combination of MoVo-LISA and MoVoApp improve cognitive mediators of behavioral change (e.g., self-efficacy or action planning), and health-related variables (e.g., pain intensity, depression) in patients with musculoskeletal disorders more than the same face-to-face group-based intervention without subsequent MoVo-App use?

3. Which cognitive and sociodemographic factors moderate and mediate the effects of MoVo-LISA and MoVo-App?

\section{Methods/design}

\section{Study design}

This study was registered on the World Health Organization International Clinical Trials Registry Platform via the German Clinical Studies Trial Register on 18 October 2018 (DRKS00014814). For an overview of the study schedule, enrollment, interventions, and assessments, see the Standard Protocol Items: Recommendations for Interventional Trials (SPIRIT) [38] schedule in Fig. 1. The SPIRIT checklist [38] can be found in Additional file 1.

This is a multicenter, pragmatic RCT of parallel design with an allocation ratio of 1:1. In this RCT, we aim to evaluate whether the MoVo-App in combination with the group-based face-to-face intervention MoVo-LISA during orthopedic rehabilitation can effectively support inactive individuals to initiate physical activity, to increase their amount of physical activity (minutes per week), and to maintain this level of physical activity (physically active: yes/no and minutes per week) in the long term.

All participants receive treatment as usual (TAU) and the face-to-face group-based intervention MoVo-LISA during their clinic stay (see Fig. 1 and the Consolidated Standards of Reporting Trials (CONSORT) flowchart in Fig. 2). After participating in the face-to-face MoVoLISA program in the clinic, the IG receives the MoVoApp at clinic discharge as a blended intervention. Following eligibility screening by the clinic staff, participants give informed consent and complete baseline outcome assessments prior to randomization and allocation (T0). Assessments are repeated at clinic discharge (T1), at 6 weeks (T2), and at 4 months after discharge (after the end of the MoVo-App and thus post-treatment) (T3), as well as 6 (T4) and 12 months (T5) after discharge (follow-up). All assessments are conducted through online questionnaires. The first two assessments (T0 and T1) take place in the clinic, and the participants are supported by a study assistant. All further online questionnaires are sent to the participants via e-mail. If the participants do not complete the questionnaires, they will be reminded after $2,4,6$, and about 14 days after receiving the questionnaire. The first and second reminders are sent to the participants by e-mail. The third reminder is carried out via telephone. If the patients cannot be reached by telephone, they are called a second time.

We are conducting and reporting the RCT in accordance with the CONSORT 2010 statement [39], the supplement of the CONSORT statement for pragmatic effectiveness trials [40], and current guidelines for executing and reporting eHealth research [41]. All procedures are approved by the ethics committee of the Albert Ludwig University of Freiburg (no. 270/18).

In a pilot study from October until December 2018, the procedures for recruitment and intervention delivery in the clinics were tested and refined. During this time, 34 participants were recruited. On the basis of experiences and feedback of patients as well as clinic staff regarding conduction and implementation of the study, some adjustments and improvements were made to 


\begin{tabular}{|c|c|c|c|c|c|c|c|c|c|c|}
\hline Timepoint & Measurement & Rater & Enrolment & Allocation & T0 & T1 & $\mathrm{T} 2$ & T3 & T4 & T5 \\
\hline Eligibility screen & & & $\mathrm{x}$ & & & & & & & \\
\hline Informed consent & & & $x$ & & & & & & & \\
\hline Allocation & & & & $\mathrm{x}$ & & & & & & \\
\hline \multicolumn{11}{|c|}{ Interventions } \\
\hline Treatment as usual (orthopedic rehabilitation) & & & & $x-$ & & $-x$ & & & & \\
\hline MoVo-LISA & & & & $x$ & 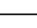 & 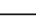 & $-x$ & & & \\
\hline MoVo-App & & & & & & $x-$ & & $-x$ & & \\
\hline \multicolumn{11}{|c|}{$\begin{array}{c}\text { Assessments } \\
\text { Inclusion/exclusion criteria }\end{array}$} \\
\hline Age $\geq 18$ years & Screening & Physician & $\bar{x}$ & & & & & & & \\
\hline Sufficient knowledge of the German language & Screening & Physician & $\mathrm{x}$ & & & & & & & \\
\hline Musculoskeletal diagnosis (ICD-10) & Medical report & Physician & $\mathrm{x}$ & & & & & & & \\
\hline Smartphone and internet access & Screening & Physician & $\mathrm{x}$ & & & & & & & \\
\hline Physically inactive & Screening & Physician & $\mathrm{x}$ & & & & & & & \\
\hline Rehabilitation following inpatient clinic treatment & Medical report & Physician & $\mathrm{x}$ & & & & & & & \\
\hline Medically unsuitable & Screening & Physician & $\mathrm{x}$ & & & & & & & \\
\hline \multicolumn{11}{|c|}{ Primary outcome } \\
\hline Physical activity & BSA subscale sport activity & Self-rating & & & $\mathrm{X}$ & & $\mathrm{x}$ & $\mathrm{x}$ & $\mathrm{x}$ & $\mathrm{x}$ \\
\hline \multicolumn{11}{|c|}{ Secondary outcomes } \\
\hline Movement activity & BSA subscale movement activity & Self-rating & & & $\mathrm{X}$ & & $\mathrm{X}$ & $\mathrm{x}$ & $\mathrm{X}$ & $\mathrm{x}$ \\
\hline \multirow[t]{7}{*}{ Cognitive predictors of behavior modification } & Sport-related & & & & & & & & & \\
\hline & - Self-efficacy & & & & $x$ & $\mathrm{x}$ & $x$ & $x$ & $x$ & $x$ \\
\hline & - Goal intention & & & & $\mathrm{x}$ & $\mathrm{x}$ & $\mathrm{x}$ & $x$ & $\mathrm{x}$ & $\mathrm{x}$ \\
\hline & - Self-concordance & Self-rating & & & $\mathrm{x}$ & $\mathrm{x}$ & $\mathrm{x}$ & $\mathrm{x}$ & $\mathrm{x}$ & $\mathrm{x}$ \\
\hline & - Barriers und barrier management & & & & $\mathrm{x}$ & $x$ & $\mathrm{x}$ & $x$ & $\mathrm{x}$ & $\mathrm{x}$ \\
\hline & - Consequence experience & & & & $x$ & $x$ & $x$ & $x$ & $x$ & $x$ \\
\hline & - Action planning & & & & $x$ & $x$ & $x$ & $x$ & $x$ & $x$ \\
\hline Pain intensity & Numeric rating scale & Self-rating & & & $\mathrm{x}$ & $\mathrm{x}$ & $\mathrm{x}$ & $\mathrm{x}$ & $\mathrm{x}$ & $\mathrm{x}$ \\
\hline Pain impairment & Brief Pain Inventory (BPI) & Self-rating & & & $\mathrm{x}$ & $x$ & $\mathrm{x}$ & $x$ & $\mathrm{x}$ & $\mathrm{x}$ \\
\hline Depression & Patient Health Questionnaire-8 (PHQ-8) & Self-rating & & & $x$ & $x$ & $x$ & $x$ & $x$ & $x$ \\
\hline Anxiety & $\begin{array}{l}\text { Generalised Anxiety Disorder Screener-7 } \\
\text { (GAD-7) }\end{array}$ & Self-rating & & & $x$ & $x$ & $x$ & $x$ & $x$ & $x$ \\
\hline Satisfaction with MoVo-App & $\begin{array}{l}\text { Adapted Items from Client Satisfaction } \\
\text { Questionnaire (CSQ- } 8)\end{array}$ & Self-rating & & & & & & $x$ & & $x$ \\
\hline App adherence & Usage data of MoVo-App & & & & & & & $x$ & & $x$ \\
\hline \multicolumn{11}{|c|}{ Effect moderating factors } \\
\hline Diagnosis according to ICD-10 & Medical report & Physician & $\mathrm{X}$ & & & & & & & \\
\hline Demographic variables & Demographic questionnaire & Self-rating & & & $\mathrm{x}$ & & & & & \\
\hline
\end{tabular}

Fig. 1 Schedule of enrollment, interventions, and assessments

optimize the recruitment strategies, outcome assessments, and the usability and installation of the MoVoApp.

\section{Proposed sample size/power calculations}

In a previous group-based MoVo-LISA trial with orthopedic patients, exercise duration was $156 \mathrm{~min} /$ week immediately after the intervention, which decreased to 92 min/week at 6 months after discharge [16]. If the MoVo-App is able to stabilize the post-treatment effect, a medium effect size of (standardized mean difference) $d$ $=0.62$ for the main effect and a small to medium effect size for the interaction effect group (MoVo-LISA + MoVo-App) $\times$ time of $d=0.35$ at the 6-month follow-up (T4) can be expected. However, the effect size estimate for the interaction effect could only be extrapolated from interaction effects observed for group trials comparing MoVo-LISA with a wait-list control group [19]. We assume a baseline measure of the outcome to be used as a covariate and a correlation between pre- and postmeasures of 0.33 based on prior data. With a power of $80 \%$, a significance level of $5 \%$, and small to moderate effect sizes, and considering an estimated dropout rate of $15 \%$, a total sample size of 224 is required.

\section{Recruitment}

Recruitment started in January 2019 and ended in December 2019. Although this study protocol was submitted for publication after the start of recruitment (due to usual writing, commenting, and approval procedures involving all coauthors), clinical trial registration took place prior to recruitment start. Participants were recruited in two orthopedic rehabilitation clinics located in southwestern Germany. In Germany, patients with severe injuries and chronic musculoskeletal conditions have the opportunity to receive rehabilitation therapies for 3 or more weeks in an orthopedic inpatient rehabilitation clinic covered by insurance companies. The target population in this study consists of persons with musculoskeletal conditions (e.g., arthritis, chronic back pain). Participants did not receive compensation for their participation. All consecutive patients are screened by a physician for eligibility upon arrival during their first medical examination. The first participant was enrolled on 7 January 2019, and the last patient was enrolled on 3 December 2019.

\section{Inclusion criteria}

Patients are included if they fulfill the following inclusion criteria: (1) age 18 years or older; (2) 


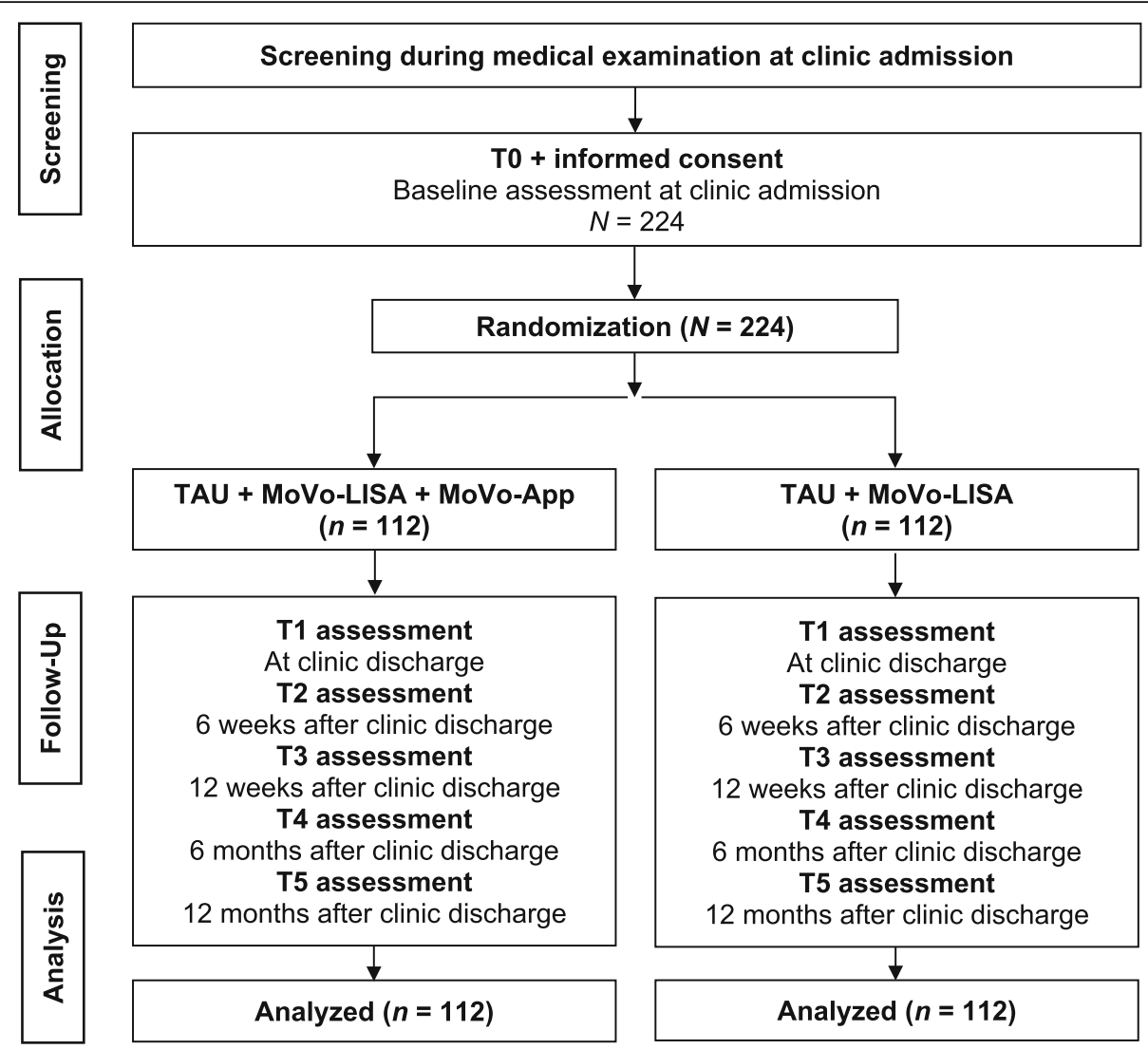

Fig. 2 Consolidated Standards of Reporting Trials (CONSORT) flowchart

musculoskeletal diagnosis classified in chapter $M$ of the International Statistical Classification of Diseases and Related Health Problems, 10th Revision (ICD-10) [42], diagnosed by a physician; (3) sufficient knowledge of the German language; (4) ownership of a smartphone and internet access for online assessments; and (5) being physically inactive (sport activity $<30 \mathrm{~min} /$ week) in the last 3 months. Exclusion criteria are (1) rehabilitation following inpatient clinic treatment (e.g., due to surgery) and (2) not able to exercise for medical reasons. Eligible patients receive an invitation to participate in the study, an information letter/leaflet, and the informed consent form and complete the baseline online questionnaire (T0) during a study information meeting with a study assistant.

\section{Randomization}

Randomization and allocation of participants to the two groups were done in advance by an independent researcher (CR) by means of a web-based program (https://www.sealedenvelope.com/) using permuted block randomization with variable block sizes of 2,4 , and 6 (randomly arranged) and a ratio of 1:1. Participants are stratified by center. The centers involved are two orthopedic clinics in southwestern Germany where recruitment is conducted consecutively. Participants are given randomly generated treatment allocations within sealed opaque envelopes. Once a participant is consented to participate in the study, the envelope is opened, and the patient is then offered the allocated treatment regimen. An independent researcher $(\mathrm{CR})$ prepares the envelopes and is responsible for the allocation of participants to trial arms.

\section{Content of the face-to-face group intervention MoVo-LISA} The psychological group program MoVo-LISA (Motivation-Volition concept to support Lifestyle-Integrated Sports Activity; three to eight patients per group) is based on both motivation theories of health behavior [43-45] and volition theories of action planning and action control [46-48]. The theoretical framework underlying the MoVo-LISA intervention program is the MoVo process model. Its core idea is the differentiation between motivational and volitional strategies [21]. Motivational strategies are used to help form a strong and self-concordant goal intention. Volitional strategies are needed to implement competencies and action control [13]. MoVo-LISA is conceptualized as a short and economical psychological program based on a standardized curriculum. It has been developed specifically for an 
inpatient rehabilitation setting and has been shown to be effective in orthopedic patients $[16,17,20]$. In this trial, MoVo-LISA is offered to all randomized participants in both the intervention and control groups by the physiotherapists or sport and exercise therapists in each clinic. Before recruitment, all MoVo-LISA instructors were trained in the standardized program by the scientific project team during a 2-day course. In addition, a kickoff meeting was organized for all clinic staff in order to inform them about the study and to familiarize them with the MoVo concept.

The program MoVo-LISA consists of two group sessions and one individual session in which patients learn how to integrate physical activity into their daily lives. It does not consist of actual physical exercise but focuses on teaching the patients how to get and stay physically active after inpatient rehabilitation. The patients prepare specific activity plans for implementation in their everyday lives based on their individual needs and living context. Hence, the activity plans of the participants vary highly from aerobics to stretching and stabilizing exercises and are based on the recommendations that were developed during their orthopedic rehabilitation. In addition, they identify barriers that discourage them from being active and develop individual strategies to cope with those barriers. After discharge from the clinic, participants receive a postal reminder and a telephone interview. A detailed description is given by Fuchs and Göhner [16, 49]. The five main components of MoVoLISA are listed in Table 1.

\section{MoVo-App}

The MoVo-App is based on the MoVo process model and was developed by a multidisciplinary team of psychologists, sport scientists, and computer scientists from the University of Freiburg. The aim was to develop an app that can be provided to individuals after clinic discharge subsequent to the group-based face-to-face intervention MoVo-LISA. The elements of the program are implemented within the MoVo-App to support participants in maintaining their acquired strategies and to put their activity plans into action within the upcoming 12 weeks after clinic discharge. We developed the MoVo-App for smartphones running iOS or Android operating systems.

After the installation of the MoVo-App, participants in the IG receive help with entering their health goals, activity plans, major barriers, and barrier management strategies developed during the MoVo-LISA group program into the app. Over the course of 12 weeks after discharge, the MoVo-App sends regular reminders to participants regarding their activity plan prior to the scheduled activity. Participants can record on the same day of the activity plan what percentage of their plan they have achieved in order to track their progress. Each week, participants are asked to evaluate their performance in terms of their satisfaction regarding the completion of their activity plans for that week. Participants can make adjustments to their activity plans or barrier management strategies if needed. All usage data of the app will be saved in the back end of the smartphone. At the end of the 12-week period, all users will be asked to finish the intervention and upload their usage data to a sharing platform (back4app.com) via a shortcut in the app. In addition to receiving the MoVo-App, participants in the IG have unrestricted access to TAU.

\section{Control condition}

The control group receives MoVo-LISA during the clinic stay and has unrestricted access to TAU.

\section{Treatment as usual}

TAU consists of a 3-week complex interdisciplinary and multimodal rehabilitation schedule. It comprises medical, physical, and psychological therapies specifically tailored to orthopedic patients. (More information on the German system of medical inpatient rehabilitation is given by Jäckel and colleagues [50]). Because TAU after clinic discharge may vary widely, TAU in this time will not follow a specific protocol.

Table 1 Elements of the MoVo-LISA program

\begin{tabular}{|c|c|c|c|}
\hline Components & Time of event & Duration & Content/aim \\
\hline $\begin{array}{l}\text { First group } \\
\text { meeting }\end{array}$ & $\begin{array}{l}\text { Days } 1-3 \text { after clinic } \\
\text { admission }\end{array}$ & $60 \mathrm{~min}$ & To reflect on health goals and ideas for physical activity \\
\hline Homework & After first group meeting & - & To prepare an individual activity plan \\
\hline $\begin{array}{l}\text { One-to-one } \\
\text { interview }\end{array}$ & $\begin{array}{l}1 \text { week after first group } \\
\text { meeting }\end{array}$ & $10 \mathrm{~min}$ & To refine the individual activity plan \\
\hline $\begin{array}{l}\text { Second group } \\
\text { meeting }\end{array}$ & $\begin{array}{l}1-3 \text { days before clinic } \\
\text { discharge }\end{array}$ & $90 \mathrm{~min}$ & To discuss barriers and barrier management to establish an activity protocol \\
\hline Postal reminder & 3 weeks after discharge & & $\begin{array}{l}\text { To remind patients of the two activity plans and the importance of their individual barrier } \\
\text { management }\end{array}$ \\
\hline Telephone call & $\begin{array}{l}5-6 \text { weeks after } \\
\text { discharge }\end{array}$ & $10 \mathrm{~min}$ & $\begin{array}{l}\text { To check the implementation of the activity plans and barrier management and to offer } \\
\text { support in case of problems }\end{array}$ \\
\hline
\end{tabular}




\section{Assessments}

The primary outcome is the level of sport activity that is assessed using the validated Movement and Sport Activity Questionnaire (BSA-F [51]). Secondary outcomes are movement activity, cognitive predictors of behavioral change, and health-related variables. According to the MoVo process model, it is necessary to change the underlying cognitions and self-regulatory skills in order to attain long-term behavior modification. The cognitive variables postulated by the MoVo process model assessed in this trial are sport-related self-efficacy, goal intention, self-concordance, action planning, perceived barriers, and barrier management, as well as sportrelated consequence experiences. Experience of pain is considered a major health indicator among orthopedic patients. On the basis of the recommendations of the Initiative on Methods, Measurement, and Pain Assessment in Clinical Trials [52, 53], we selected pain-related outcome measures, including physical and emotional functioning. Demographic and clinical variables (e.g., age, sex, ICD-10 diagnosis), intervention adherence, and satisfaction are also assessed. The outcome measures and time points of interest are summarized in Fig. 1.

\section{Primary outcome}

The level of sport activity in minutes per week is measured with the BSA-F [51]. This is a validated German self-report instrument used to measure the level of movement activities (functional physical activities of daily living; see "Secondary outcomes" section below) and sport activities (sport- and health-related exercises, such as soccer, jogging, Nordic walking, Pilates, or fitness training) in minutes per week. For the sport activity subscale, the participants list a maximum of three sport activities in which they have engaged within the last 4 weeks. For each activity episode, participants indicate the frequency and duration in minutes. Activities that do not target larger groups of skeletal muscles and do not lead to maintenance of or increases in endurance, power, coordination, or flexibility are classified as invalid activities for this group. For each valid activity, an activity amount in minutes per week is calculated by multiplying frequency and duration divided by 4 . All single amounts of the named sport activities are added to obtain the individual's score on the Sport Activity Index. The validity of the BSA-F has been shown in several studies. However, data do not permit an analysis of the reliability of the instrument [51].

\section{Secondary outcomes}

\section{Movement activity}

Movement activity is measured with the BSA-F using the respective subscale [51]. The movement activity subscale consists of work- and leisure time-related movement activities within the last 4 weeks. Participants first rate their activity levels during work with regard to the frequency of (1) sitting (inversely coded), (2) moderate movement, and (3) intensive movement on a scale from $0=$ not at all to $3=$ a lot. Participants are then asked to indicate the duration in minutes per day and the frequency during the last 4 weeks for the eight most common movement activities in everyday life (e.g., riding a bike to work or carrying home groceries by foot). For each activity, an amount in minutes per week is calculated by multiplying frequency and duration divided by 4. These are added to obtain the individual's score on the Movement Activity Index for the work- and leisure time-related movement activities separately.

\section{Self-efficacy}

In accordance with Schwarzer and colleagues [54-56], three different types of self-efficacy will be assessed: the belief that someone is able to (1) begin regular physical exercise, (2) maintain regular activity over a longer time period, and (3) resume regular activity after interruption. Each type of self-efficacy is measured with one item; the scores of the three items will be combined into one mean value. The response format is a 6-point Likert scale ranging from $0=\mathrm{I}$ don't feel capable at all to $5=\mathrm{I}$ feel 100 per cent capable. An earlier study [15] yielded a satisfactory internal consistency ranging from $\alpha=0.75$ to $\alpha=0.92$ at different time points.

\section{Goal intention}

The strength of goal intention is assessed with a single item: "How strong is your intention to exercise regularly within the following weeks and months?" Participants can respond on a 6-point Likert scale ranging from $0=\mathrm{I}$ do not intent to exercise regularly to $5=\mathrm{I}$ do have a strong intention to exercise regularly.

\section{Self-concordance}

Self-concordance is measured using the German SSK scale (Sport-und bewegungsbezogene Selbstkonkordanz) [57], consisting of 12 items based on the self-concordance model of Sheldon and Elliot [45]. The SSK scale involves four subscales that measure the intrinsic, identified, introjected, and extrinsic motivation for being physically active. Only participants who indicated having at least a weak exercise-related goal intention (strength of goal intention $>1$ ) are receiving this questionnaire. The 4-point Likert scale ranges from $1=$ not true to $4=$ true.

This instrument has proved to be a reliable and valid measure of exercise-related self-concordance [58]. In two separate studies reported by Seelig and Fuchs [58], the reliability of the subscales ranged from $\alpha=0.70$ (identified motivation) to $\alpha=0.82$ (intrinsic motivation). 
Evidence of validity was provided by a correlation of $r=$ 0.47 between the SSK scale and the intention to engage in physical exercise [58].

\section{Barriers and barrier management}

The two scales of sport-related situational barriers and sport-related barrier management [59] allow detailed analyses of the process of volitional action control in the field of sport and physical activity. The questionnaire to assess perceived barriers contains 13 items presenting the most common sport-related situational barriers. Participants are asked how often these barriers discourage them from engaging in sport activity. The questionnaire assessing strategies for barrier management contains 15 items assessing the most common barrier management strategies. In this questionnaire, participants are asked how often they use these strategies. In both questionnaires, participants rate the items on a 4-point Likert scale ranging from $1=$ almost never to $4=$ almost always. Both scales are reliable (sport-related barrier management: $\alpha=0.78$, situational barriers: $\alpha=0.81$ ) and valid instruments [59].

\section{Consequence experience}

Expectation about sport-related consequence experience is assessed using an instrument developed and validated by Fuchs [60]. It includes 16 positive and negative versions of outcome experience of physical exercise. Participants are asked to respond on a 4-point Likert scale ranging from $1=$ not true to $4=$ true. The positive and negative experience items are summed separately. The difference of positive-negative items is derived to provide an outcome experience index [60].

\section{Action planning}

Participants are asked whether they already know which physical exercise to perform in the following weeks and months. If the answer is "yes," patients will be asked to note this activity. An opportunity is provided to name a second activity. For each of these activities, participants are asked whether they already know (1) when and (2) where they will perform it, (3) how they will get there, and (4) how often and (5) with whom they will perform the activity. An action planning score is formed by summing the number of positive answers (including naming the activity plus planning details).

\section{Pain intensity}

We are using an 11-point numerical rating scales for rating the worst, least, and average pain during the last week as well as the current pain level, and the mean of the four scales is calculated. The participants evaluate their pain from 0 to 10 , with 0 meaning "no pain" and 10 meaning "pain as bad as you can imagine." Dworkin and colleagues reported the internal consistency of a composite pain score averaging ratings of current pain, average pain, and worst pain as satisfactory to high $(\alpha=$ $0.77)$. The composite score was normally distributed, contrary to the individual ratings [61].

\section{Pain interference}

The Brief Pain Inventory (BPI) allows participants to rate the degree to which their pain interferes with common aspects of emotional and physical function $[62,63]$. The seven BPI interference items assess pain interference during the past $24 \mathrm{~h}$ on an 11-point scale from 0 ("does not interfere") to 10 ("completely interferes"). The BPI is a reliable $(\alpha=0.88)$ and valid measure of the interference of pain with physical and emotional functioning [64].

\section{Depression}

The eight-item Patient Health Questionnaire depression scale [65] is a valid diagnostic measure of the severity of depressive symptoms based on the Diagnostic and Statistical Manual of Mental Disorders, Fifth Edition (DSM-5). With the exception of the symptom of suicidal ideation or self-injurious thoughts, each of the nine DSM-5 criteria for major depression can be scored from 0 ("not at all") to 3 ("nearly every day") in the past 2 weeks. The instruments shows satisfactory validity and reliability [66].

\section{Anxiety}

The seven-item Generalized Anxiety Disorder scale [67] describes the most prominent diagnostic features of the DSM- 5 criteria A, B, and C for generalized anxiety disorder. These can be scored from 0 ("not at all") to 3 ("more than half the days") during the last 2 weeks. Its reliability and validity is good [65] with excellent internal consistency $(\alpha=0.92)$ and good test-retest reliability (intraclass correlation coefficient, 0.83).

\section{Intervention adherence}

The attrition rate (percentage of individuals who no longer use the app, as assessed by their log-on data) gives an estimate of the individuals' intervention adherence. Also, usage time will be assessed for every user who will upload the app at the end of the intervention.

\section{Patient satisfaction}

The Client Satisfaction Questionnaire [68, 69] has been optimized for the assessment of client satisfaction with internet interventions (Client Satisfaction Questionnaire adapted to Internet-based interventions [CSQ-I] [70]). We adapted this measure for the use of apps by replacing the word "training" with "app." The CSQ-I is a 
valid and reliable ( $\alpha$ between 0.93 and 0.95 ) measure of client satisfaction [70].

\section{Statistical analyses}

The primary outcome of physical activity assessed with the respective subscale of the BSA-F at T4 (post-treatment of MoVo-App) will be analyzed as a dependent variable and its baseline value as a covariate, adjusted for sex and age and potentially other covariates. Continuous secondary outcomes will be analyzed accordingly. The statistical analysis for all research questions will be based on linear mixed-effects models with restricted maximum likelihood estimation to account for missing data [71]. The model is a mixture model, thus assuming two distinct distributions: (1) a binomial model (patients becoming physically active from inactivity and vice versa) and (2) a Gaussian distribution of minutes of physical activity per week for those being physically active. In the linear mixed-effects models, time (T1 to T5) will be the within-subjects factor, and intervention (MoVo-LISA and TAU plus MoVo-App vs. MoVo-LISA and TAU) will be the between-subjects factor. All two-way interactions will be considered. Main effects and interactions will be estimated for time points $\mathrm{T} 3$ to $\mathrm{T} 5$.

Baseline minutes of sport activity, age, sex, and body mass index will be included as covariates, along with other relevant demographic and clinical characteristics (e.g., disease, pain intensity, pain interference) collected through patient self-report. The model will allow for random intercepts, and all other factors will be treated as fixed effects. All statistical analyses will be performed using R software [72].

We expect to see a skewed distribution in our primary outcome physical activity, and, following guidance [72, 73], we will use transformations or consider using generalized or mixture-distribution mixed models [72]. Effects will be considered significant if $p<0.05$. Assumptions for general, generalized, or mixture-distribution models will be checked graphically and with tests appropriate to the model $[74,75]$. In addition, we will run several model checks by comparing increasingly complex models with different covariance structures, link functions, and estimation procedures with a null model via likelihood ratio tests and comparison of fit via the Akaike information criterion and Schwartz's Bayesian information criterion [76].

To assess the mediating and moderating effects of psychosocial factors, the linear mixed-effects model of repeated measures will be extended by including psychosocial factors as random effect covariates. Their added effect as covariates, their interaction with IG, and a three-way interaction with IG and time will be estimated. It will be considered whether and how psychosocial intermediary effects depend on the intervention. Effects will be considered significant if $p<0.05$.

The analysis does not consider a cluster effect of using two sites for the intervention. This decision is based on the high standardization in the delivery of the intervention and all study procedures in both sites. Differences in patient population between clinics will be accounted for by including demographic and clinical variables as covariates in the analysis. Furthermore, clinic was also used as a stratification factor in the randomization scheme (as detailed above).

Standardized mean differences and 95\% confidence intervals will be calculated to measure the between-group effect size at post-treatment (T3) and follow-up (T4, T5). All analyses will be performed on an intention-totreat basis. Completer (per-protocol) analyses will be conducted to investigate the influence of study attrition on study results. In addition, we will analyze the extent to which participants adhere to physical activity in accordance with the National Recommendations for Physical Activity and Physical Activity Promotion [77].

\section{Ethical considerations}

Participants eligible for the study receive detailed study information and are asked for their participation (informed consent). Smartphone-based interventions have proved to be effective in a variety of RCTs without clinically meaningful side effects being reported yet $[28,78]$. Due to the focus on patients with musculoskeletal conditions, the participants are at an increased risk of injury compared with the general population. The medical suitability of participants for the intervention is recorded continuously as part of the inpatient rehabilitation diagnostic assessment and treatment. Data security and confidentiality are guaranteed under the General Data Protection Regulation Act. A data security concept is created together with the data security department at the University of Freiburg, Germany. Central ethical approval has been confirmed from the Albert Ludwig University of Freiburg (DRKS00014814).

\section{Source of monetary or material support}

The study is supported via the institutional budget with no external funding. There are no sponsors involved in this study.

\section{Discussion}

The present trial focuses on individuals with musculoskeletal conditions. The purpose of the MoVo-App trial is to evaluate a blended intervention of inpatient, faceto-face treatment coupled with a mobile app to help initiate and promote the maintenance of physical activity in this vulnerable patient group. Previous studies have 
shown that the initial adoption of physical activity is possible and successful in this population. Most studies to date have not succeeded in implementing long-term habit change in participants.

In a rigorous evaluation, this trial addresses some of the gaps of prior studies. First, given that many people have busy lifestyles, they value convenient access to health behavior change programs [79]. In this context, the MoVo-App offers the opportunity to support behavioral interventions in real-life situations. Second, although apps have a high potential to support individuals to become active more sustainably, most of the apps are lacking evidence-based content [80]. In the development of the MoVo-App, we systematically translated the theoretical concepts of the MoVo process model into the app. Third, outcomes in this study not only comprise physical activity behavior but also measure health-related variables (e.g., pain intensity, depression) and underlying psychological mediators of behavior change as postulated within the MoVo concept. This will allow a deeper understanding of the treatment processes within MoVo-LISA and the MoVo-App, which in turn can help to further develop the intervention. Fourth, by implementing the intervention directly into routine healthcare, we will be able to enroll a more naturalistic sample. Most trials of internet- and mobile appbased interventions suffer from a selection bias with mostly highly educated and female participants selfselecting into these interventions $[35,81]$. Due to the consecutive routine care sample, self-selection bias can be minimized in this trial. Finally, MoVo-LISA is conceptualized to be used for a wide range of settings and indications. Therefore, the blended intervention of MoVo-LISA and the MoVo-App can easily be transferred to other (healthcare) settings as well as health conditions.

Some limitations need to be addressed. First, the primary outcome is assessed with a questionnaire that is only available in German and does not provide a minimal clinically important difference. Second, there are no objective measures of the primary outcome physical activity conducted. If the results of this study indicate that the blended intervention of MoVo-LISA and MoVo-App is more effective than MoVo-LISA alone, its clinical impact on the promotion and maintenance of an increased level of physical activity could be substantial.

\section{Trial status}

This is protocol version number 3, dated June 04, 2020. Recruitment began on 7 January 2019; the last patient was enrolled on 3 December 2019. The first results of this study are expected in 2021.

\section{Supplementary information}

Supplementary information accompanies this paper at https://doi.org/1 0.1186/s13063-020-04438-4

Additional file 1. SPIRIT 2013 Checklist: Recommended items to address in a clinical trial protocol and related documents*.

\begin{abstract}
Abbreviations
BPI: Brief Pain Inventory; BSA-F: Movement and Sport Activity Questionnaire; CONSORT: Consolidated Standards of Reporting Trials; CSQ-I: Client Satisfaction Questionnaire adapted to Internet-based interventions; DRKS: German Clinical Studies Trial Register; ICD-10: International Statistical Classification of Diseases and Related Health Problems, 10th Revision; IG: Intervention group; MoVo: Motivation-Volition concept; MoVoLISA: Motivation-Volition concept to support Lifestyle-Integrated Sports Activity; MoVo-App: MoVo-based smartphone application; RCT: Randomized controlled trial; SPIRIT: Standard Protocol Items: Recommendations for Interventional Trials; TAU: Treatment as usual
\end{abstract}

\section{Acknowledgements}

We thank Dr. Hans-Albrecht Kulenkampff of the "Schwarzwaldklinik" orthopedic clinic and Raimund Weber, Dr. Karin Bagheri-Fam, Gabriele StipplerStrobel, and Silke Hellhake of the "Breisgau Klinik" orthopedic clinic for their support in the recruitment of the participants. Special thanks go to all staff in clinics contributing to recruitment and data collection and to the physiotherapists, health therapists, and sport therapists in the clinics who conduct the MoVo-LISA intervention. We thank Abdullah Khan, Victor Vadakechirayath George, and Ji Qi for their work in developing the MoVo-App. Moreover, we thank our study assistants Léa Ouedraogo and Nicole Kulawinski and all interns and students for their support in the development of the intervention and in conducting the study.

\section{Authors' contributions}

$J \mathrm{~L}, \mathrm{RF}$, and RW initiated this study. All authors contributed to the design of this study. JL, RF, WG, and RW developed the intervention content. JL, RF, RW, and CR developed the assessments. RW, SP, VH, RF, JL, and SK are responsible for recruitment. $\mathrm{RW}, \mathrm{SP}, \mathrm{VH}$, and $\mathrm{J}$ are responsible for study administration. CR is responsible for randomization and allocation. JL wrote the draft of the manuscript. All authors contributed to the further writing of the manuscript, and all authors read and approved the final version of the manuscript.

\section{Funding}

The authors received no specific funding for their work on this project. The article-processing charge was funded by the German Research Foundation (DFG) and the University of Freiburg in the Open Access Publishing funding program.

\section{Availability of data and materials}

The final dataset, excluding any demographic and personal information allowing identification of participants, and the statistical analysis code will be accessible from the corresponding author upon reasonable request.

\section{Ethics approval and consent to participate}

Central ethical approval has been confirmed by Albert Ludwig of University of Freiburg (DRKS00014814), and we will not begin recruiting at other centers in the trial until local ethical approval has been obtained. All participants give their written consent to participate on a voluntary basis and can withdraw from the study at any time without giving reasons and without their decision affecting their medical care.

\section{Consent for publication}

Not applicable, because the study does not contain individual data.

\section{Competing interests}

The authors declare that they have no competing interests.

\section{Author details}

${ }^{1}$ Department of Psychiatry and Psychotherapy Medical Center, Freiburg, Germany. ${ }^{2}$ Department of Sport Psychology, Institute of Sports and Sport 
Science, University of Freiburg, Schwarzwaldstrasse 175, 79117 Freiburg, Germany. ${ }^{3}$ Schwarzwaldkliniken Bad Krozingen, Bad Krozingen, Germany. ${ }^{4}$ Department of Health Psychology, Catholic University of Applied Sciences, Freiburg, Germany.

Received: 30 September 2019 Accepted: 20 May 2020 Published online: 08 June 2020

\section{References}

1. Hoy D, Bain C, Williams G, March L, Brooks P, Blyth F, et al. A systematic review of the global prevalence of low back pain. Arthritis Rheum. 2012; 64(6):2028-37.

2. Farioli A, Mattioli S, Quaglieri A, Curti S, Violante FS, Coggon D. Musculoskeletal pain in Europe: the role of personal, occupational, and social risk factors. Scand J Work Environ Health. 2014;40(1):36-46.

3. Barbour KE, et al. Prevalence of doctor-diagnosed arthritis and arthritisattributable activity limitation-United States, 2010-2012. MMWR Morb Mortal Wkly Rep. 2013;62(44):869-73.

4. Schmidt CO, Günther KP, Goronzy J, Albrecht K, Chenot JF, Callhoff J, et al. Frequencies of musculoskeletal symptoms and disorders in the populationbased German National Cohort (GNC) [in German]. Bundesgesundheitsblatt Gesundheitsforschung Gesundheitsschutz. 2020;63(4):415-25.

5. Hagen KB, Dagfinrud H, Moe RH, Østerås N, Kjeken I, Grotle M, et al. Exercise therapy for bone and muscle health: an overview of systematic reviews. BMC Med. 2012;10:167.

6. Weinstein SI, Yelin EH, Watkins-Castillo SI. The burden of musculoskeletal diseases in the United States: prevalence, societal and economic cost. 3rd ed. Rosemont: United States Bone and Joint Initiative; 2015. https://www. boneandjointburden.org/docs/The\%20Burden\%20of\%20Musculoskeletal\%2 ODiseases\%20in\%20the\%20United\%20States\%20(BMUS)\%203rd\%2 OEdition\%20(Dated\%2012.31.16).pdf. Accessed 4 June 2020.

7. Vos T, Abajobir AA, Abate KH, Abbafati C, Abbas KM, Abd-Allah F, et al. Global, regional, and national incidence, prevalence, and years lived with disability for 328 diseases and injuries for 195 countries, 1990-2016: a systematic analysis for the Global Burden of Disease Study 2016. Lancet. 2017;390(10100):1211-59

8. National Institute for Health and Care Excellence (NICE). Osteoarthritis: care and management. Clinical guideline CG177. London: NICE; 2014. https:// www.nice.org.uk/guidance/cg177. Accessed 4 June 2020.

9. Scottish Intercollegiate Guidelines Network (SIGN). SIGN 136: Management of chronic pain. Edinburgh: SIGN; 2013. https://www.sign.ac.uk/assets/ sign136.pdf. Accessed 4 June 2020.

10. Geneen LJ, Smith BH, Clarke C, Martin D, Colvin LA, Moore RA. Physical activity and exercise for chronic pain in adults: an overview of Cochrane Reviews. Cochrane Database Syst Rev. 2017;1 (1):CD011279.

11. Jack K, McLean SM, Moffett JK, Gardiner E. Barriers to treatment adherence in physiotherapy outpatient clinics: a systematic review. Man Ther. 2010:15(3):220-8.

12. Physical Activity Guidelines Advisory Committee. Physical Activity Guidelines Advisory Committee scientific report. Washington, DC: U.S. Department of Health and Human Services; 2018.

13. Fuchs R. Das MoVo-Modell als theoretische Grundlage für Programme der Gesundheitsverhaltensänderung. In: Fuchs R, Göhner W, Seelig H, editors. Aufbau eines körperlich-aktiven Lebensstils. Göttingen: Hogrefe; 2007. p. $317-25$.

14. Fuchs R. Das Motivations-Volitions-Konzept. Public Health Forum. 2013;21 (2): 32 e1-e3.

15. Göhner W, Dietsche C, Fuchs R. Increasing physical activity in patients with mental illness - a randomized controlled trial. Patient Educ Couns. 2015; 98(11):1385-92.

16. Fuchs R, Goehner W, Seelig H. Long-term effects of a psychological group intervention on physical exercise and health: the MoVo concept. J Phys Act Health. 2011;8(6):794-803.

17. Göhner W, Seelig H, Fuchs R. Intervention effects on cognitive antecedents of physical exercise: a 1-year follow-up study. Appl Psychol Health Well Being. 2009; 1(2):233-56.

18. Gerber M, Fuchs R, Pühse U. Einfluss eines Kurz-Interventionsprogramms auf das Bewegungsverhalten und seine psychologischen Voraussetzungen bei Übergewichtigen und Adipösen. Z Gesundheitspsychologie. 2010;18(4):159-69.
19. Wurst R, Kinkel S, Lin J, Göhner W, Fuchs R. Promoting physical activity through a psychological group intervention in cardiac rehabilitation: a randomized controlled trial. J Behav Med. 2019;42(6):1104-16.

20. Fuchs R, Göhner W, Seelig H, Fleitz A, Mahler C, Schittich I. Lebensstilintegrierte sportliche Aktivität: Ergebnisse der MoVo-LISA Interventionsstudie. B\&G Bewegungstherapie und Gesundheitssport. 2010; 26(6):270-6.

21. Fuchs R, Seelig H, Göhner W, Burton NW, Brown WJ. Cognitive mediation of intervention effects on physical exercise: causal models for the adoption and maintenance stage. Psychol Health. 2012;27(12):1480-99.

22. Dallinga JM, Mennes M, Alpay L, Bijwaard H, Baart de la Faille-Deutekom M. App use, physical activity and healthy lifestyle: a cross sectional study. BMC Public Health. 2015:15:833.

23. Bort-Roig J, Gilson ND, Puig-Ribera A, Contreras RS, Trost SG. Measuring and influencing physical activity with smartphone technology: a systematic review. Sports Med. 2014;44(5):671-86.

24. Fanning J, Mullen SP, McAuley E. Increasing physical activity with mobile devices: a meta-analysis. J Med Internet Res. 2012;14(6):e161.

25. Ljótsson B, Atterlöf E, Lagerlöf $M$, Andersson E, Jernelov $S$, Hedman $E$, et al. Internet-delivered acceptance and values-based exposure treatment for fibromyalgia: a pilot study. Cogn Behav Ther. 2014;43(2):93-104.

26. Middelweerd A, Mollee JS, van der Wal CN, Brug J, Te Velde SJ. Apps to promote physical activity among adults: a review and content analysis. Int Behav Nutr Phys Act. 2014;11:97.

27. Goode AD, Reeves MM, Eakin EG. Telephone-delivered interventions for physical activity and dietary behavior change. Am J Prev Med. 2012;42(1):81-8,

28. Schoeppe S, Alley S, van Lippevelde W, Bray NA, Williams SL, Duncan MJ, et al. Efficacy of interventions that use apps to improve diet, physical activity and sedentary behaviour: a systematic review. Int I Behav Nutr Phys Act. 2016;13(1):127.

29. Davies CA, Spence JC, Vandelanotte C, Caperchione CM, Mummery WK. Meta-analysis of internet-delivered interventions to increase physical activity levels. Int J Behav Nutr Phys Act. 2012;9(1):52.

30. Eysenbach G. The law of attrition. J Med Internet Res. 2005;7(1):e11.

31. Chiu TM, Eysenbach G. Stages of use: consideration, initiation, utilization, and outcomes of an internet-mediated intervention. BMC Med Inform Decis Mak. 2010;10:73.

32. Lillevoll KR, Vangberg HCB, Griffiths KM, Waterloo K, Eisemann MR. Uptake and adherence of a self-directed internet-based mental health intervention with tailored e-mail reminders in senior high schools in Norway. BMC Psychiatry. 2014;14:14.

33. Donkin L, Christensen H, Naismith SL, Neal B, Hickie IB, Glozier N. A systematic review of the impact of adherence on the effectiveness of etherapies. J Med Internet Res. 2011;13(3):e52.

34. Kelders SM, Kok RN, Ossebaard HC, Van Gemert-Pijnen JE. Persuasive system design does matter: a systematic review of adherence to web-based interventions. J Med Internet Res. 2012;14(6):e152.

35. Lin J, Faust B, Ebert DD, Krämer L, Baumeister H. A web-based acceptancefacilitating intervention for identifying patients' acceptance, uptake, and adherence of internet- and mobile-based pain interventions: randomized controlled trial. J Med Internet Res. 2018;20(8):e244 A published correction appears in J Med Internet Res. 2019;21(2):e12015.

36. Cuijpers P, Sijbrandij M, Koole SL, Andersson G, Beekman AT, Reynolds CF. The efficacy of psychotherapy and pharmacotherapy in treating depressive and anxiety disorders: a meta-analysis of direct comparisons. World Psychiatry. 2013;12(2):137-48

37. Erbe D, Eichert HC, Riper H, Ebert DD. Blending face-to-face and internetbased interventions for the treatment of mental disorders in adults: systematic review. J Med Internet Res. 2017;19(9):e306.

38. Chan AW, Tetzlaff JM, Gøtzsche PC, Altman DG, Mann H, Berlin JA, et al. SPIRIT 2013 explanation and elaboration: guidance for protocols of clinical trials. BMJ. 2013;346:e7586.

39. Moher D, Hopewell S, Schulz KF, Montori V, Gøtzsche PC, Devereaux PJ, et al. CONSORT 2010 explanation and elaboration: updated guidelines for reporting parallel group randomised trials. BMJ. 2010;340:C869.

40. Zwarenstein M, Treweek S, Gagnier JJ, CONSORT group. Pragmatic Trials in Healthcare (Practihc) group. Improving the reporting of pragmatic trials: an extension of the CONSORT statement. BMJ. 2008;337:a2390.

41. Eysenbach G, CONSORT-EHEALTH Group. CONSORT-EHEALTH: improving and standardizing evaluation reports of Web-based and mobile health interventions. J Med Internet Res. 2011;13(4):e126. 
42. World Health Organization (WHO). International Statistical Classification of Diseases and Related Health Problems. 10th Revision. Geneva: WHO; 2011. www.who.int. Accessed 1 Apr 2019.

43. Ajzen I. The Theory of Planned Behavior. Organ Behav Hum Decis Process. 1991;50(2):179-211.

44. Bandura A. Health promotion from the perspective of social cognitive theory. Psychol Health. 1998;13(4):623-49.

45. Sheldon KM, Elliot AJ. Goal striving, need satisfaction, and longitudinal wellbeing: the self-concordance model. J Pers Soc Psychol. 1999;76(3):482-97.

46. Gollwitzer PM. Implementation intentions: strong effects of simple plans. Am Psychol. 1999;54(7):493-503.

47. Kuhl J. A functional-design approach to motivation and self-regulation: the dynamics of personality systems interactions. In: Boekaerts M, Pintrich PR, Zeidner M, editors. Handbook of self-regulation, editors. San Diego: Academic Press; 2000. p. 111-69.

48. Schwarzer R, Schuz B, Ziegelmann JP, Lippke S, Luszczynska A, Scholz U. Adoption and maintenance of four health behaviors: theory-guided longitudinal studies on dental flossing, seat belt use, dietary behavior, and physical activity. Ann Behav Med. 2007;33(2):156-66.

49. Göhner W, Fuchs R. Änderung des Gesundheitsverhaltens: MoVoGruppenprogramme für körperliche Aktivität und gesunde Ernährung. Göttingen: Hogrefe; 2007.

50. Jäckel $W H$, Bengel J, Herdt J. Research in rehabilitation: results from a research network in Southwest Germany. Stuttgart: Schattauer Verlag; 2006.

51. Fuchs R, Klaperski S, Gerber M, Seelig H. Messung der Bewegungs-und Sportaktivität mit dem BSA-Fragebogen. Z Gesundheitspsychologie. 2015; 23(2):60-76.

52. Turk DC, Dworkin RH, Allen RR, Bellamy N, Brandenburg N, Carr DB, et al. Core outcome domains for chronic pain clinical trials: IMMPACT recommendations. Pain. 2003;106(3):337-45.

53. Dworkin RH, Turk DC, Farrar JT, Haythornthwaite JA, Jensen MP, Katz NP et al. Core outcome measures for chronic pain clinical trials: IMMPACT recommendations. Pain. 2005;113(1-2):9-19.

54. Luszczynska A, Schwarzer R. Planning and self-efficacy in the adoption and maintenance of breast self-examination: a longitudinal study on selfregulatory cognitions. Psychol Health. 2003;18(1):93-108.

55. Sniehotta FF, Scholz U, Schwarzer R. Bridging the intention-behaviour gap: planning, self-efficacy, and action control in the adoption and maintenance of physical exercise. Psychol Health. 2005;20(2):143-60.

56. Schwarzer R, Renner B. Social-cognitive predictors of health behavior: action self-efficacy and coping self-efficacy. Health Psychol. 2000;19(5):487-95.

57. Seelig H, Fuchs R. Die sport- und bewegungsbezogene Selbstkonkordanz [Measuring sport-and movement-related self concordance]. ZEITSCHRIFT FUR SPORTPSYCHOLOGIE, 2006;13(4):121-39.

58. Seelig H, Fuchs R. Messung der sport-und bewegungsbezogenen Selbstkonkordanz. Z Sportpsychologie. 2006;13(4):121-39 https://www.sport. uni-freiburg.de/de/institut/Arbeitsbereiche/psychologie/psych_proj/ssk/ seelig-fuchs-2006.pdf.

59. Krämer L, Fuchs R. Barrieren und Barrierenmanagement im Prozess der Sportteilnahme: Zwei neue Messinstrumente. Z Gesundheitspsychologie. 2010;18(4):170-82.

60. Fuchs R. Das Motivations-Volitions-Konzept. Public Health Forum. 2013;21(2): 32-4.

61. Dworkin SF, Von Korff M, Whitney CW, Le Resche L, Dicker BG, Barlow W. Measurement of characteristic pain intensity in field research [abstract]. Pain. 1990;41 Suppl 1:S290.

62. Radbruch L, Loick G, Kiencke P, Lindena G, Sabatowski R, Grond S, et al. Validation of the German version of the Brief Pain Inventory. J Pain Symptom Manage. 1999;18(3):180-7.

63. Cleeland CS. The Brief Pain Inventory. Houston: Pain Research Group; 1991 p. $143-5$.

64. Keller S, Bann CM, Dodd SL, Schein J, Mendoza TR, Cleeland CS. Validity of the Brief Pain Inventory for use in documenting the outcomes of patients with noncancer pain. Clin J Pain. 2004;20(5):309-18.

65. Kroenke K, Strine TW, Spitzer RL, Williams JBW, Berry JT, Mokdad AH. The PHQ-8 as a measure of current depression in the general population. $J$ Affect Disord. 2009;114(1-3):163-73.

66. Pressler SJ, Subramanian U, Perkins SM, Gradus-Pizlo I, Kareken D, Kim J, et al. Measuring depressive symptoms in heart failure: validity and reliability of the patient health questionnaire-8. Am J Crit Care. 2011;20(2):146-52.
67. Löwe B, Decker O, Müller S, Brähler E, Schellberg D, Herzog W, et al. Validation and standardization of the generalized anxiety disorder screener (GAD-7) in the general population. Med Care. 2008;46(3):266-74.

68. Attkisson CC, Zwick R. The Client Satisfaction Questionnaire: psychometric properties and correlations with service utilization and psychotherapy outcome. Eval Program Plann. 1982:5(3):233-7.

69. Schmidt J, Lamprecht F, Wittmann WW. Satisfaction with inpatient management. Development of a questionnaire and initial validity studies [in German]. Psychother Psychosom Med Psychol. 1989;39(7):248-55.

70. Boß L, Lehr D, Reis D, Vis C, Riper H, Berking M, et al. Reliability and validity of assessing user satisfaction with web-based health interventions. J Med Internet Res. 2016;18(8):e234

71. West BT, Welch KB, Gałecki AT. Linear mixed models: a practical guide using statistical software. 2nd ed. Boca Raton: Chapman \& Hall/CRC; 2015.

72. R Foundation for Statistical Computing. R: a language and environment for statistical computing. Vol. 1. Vienna: R Foundation for Statistical Computing; 2008. http://www.r-project.org. Accessed 4 June 2020.

73. Baldwin SA, Fellingham GW, Baldwin AS. Statistical models for multilevel skewed physical activity data in health research and behavioral medicine. Health Psychol. 2016;35(6):552-62.

74. Zuur AF, leno EN, Elphick CS. A protocol for data exploration to avoid common statistical problems. Methods Ecol Evol. 2010;1(1):3-14.

75. Zuur AF, leno EN. A protocol for conducting and presenting results of regression-type analyses. Methods Ecol Evol. 2016;7(6):636-45.

76. King T. Longitudinal data analysis for the behavioral sciences using $\mathrm{R}$ by Jeffrey D. Long (Thousand Oaks, CA: Sage, 2012) [Pp. 542.] ISBN 1-41298268-9 £61.00. Int J Lang Commun Disord. 2016;51(3):355.

77. Rütten A, Pfeifer K, Banzer W, Ferrari N, Füzéki E, Geidl W, et al. Nationale Empfehlungen für Bewegung und Bewegungsförderung. Erlangen: FAU Erlangen-Nürnberg; 2016. https://www.sport.fau.de/files/2016/05/NationaleEmpfehlungen-für-Bewegung-und-Bewegungsförderung-2016.pdf. Accessed 4 June 2020.

78. Feter N, Dos Santos TS, Caputo EL, da Silva MC. What is the role of smartphones on physical activity promotion? A systematic review and meta-analysis. Int J Public Health. 2019;64(5):679-90.

79. Dennison L, Morrison L, Conway G, Yardley L. Opportunities and challenges for smartphone applications in supporting health behavior change: qualitative study. J Med Internet Res. 2013;15(4):e86.

80. Knight E, Stuckey MI, Prapavessis H, Petrella RJ. Public health guidelines for physical activity: is there an app for that? A review of Android and Apple app stores. JMIR Mhealth Uhealth. 2015;3(2):e43.

81. Cuijpers $P$, van Straten A, Warmerdam L, van Rooy MJ. Recruiting participants for interventions to prevent the onset of depressive disorders: possible ways to increase participation rates. BMC Health Serv Res. 2010;10:181.

\section{Publisher's Note}

Springer Nature remains neutral with regard to jurisdictional claims in published maps and institutional affiliations.

Ready to submit your research? Choose BMC and benefit from:

- fast, convenient online submission

- thorough peer review by experienced researchers in your field

- rapid publication on acceptance

- support for research data, including large and complex data types

- gold Open Access which fosters wider collaboration and increased citations

- maximum visibility for your research: over $100 \mathrm{M}$ website views per year

At $\mathrm{BMC}$, research is always in progress.

Learn more biomedcentral.com/submission 